\title{
A RESEARCH PAPER ON SELF DRIVING AUTONOUMOUS CAR
}

\author{
S Gagan Kumar, Sagarika V Sringar, Viganesha H R, \\ Student, Department of Computer Science and Engineering, \\ SJBIT, Bengaluru, 560060 \\ Shanthakumar H C \\ Associate Professor, Department of Computer Science and Engineering, \\ SJBIT, Bengaluru, 560060
}

\begin{abstract}
The concept of an autonomous car or the selfdriving car has been there for more than a century. The Ghost Car aims at achieving level 5 Autonomous Driving by implementing complex Machine Learning algorithms to detect objects, traffic signs, and lane-keep assistance of the vehicle which allow making appropriate decisions on the vehicle's movement.
\end{abstract}

\section{INTRODUCTION}

Land vehicles have been proven to be a major boon to the logistic transportation and for personal use as Land Transport provides Door to Door connectivity across the world. Many companies and projects have been existing for a long time to achieve the concept of autonomous driving.

Autonomous control implies satisfactory performance under various uncertainties in the environment and it's the ability to compensate for system failures without external intervention. The automobile industry has always been at the edge of creating a revolution by moving to self-driving vehicles, and the driving force behind is the fast-development technologies such as Internet Of Things (IoT). With IoT, billions of devices are connected online and the majority of today's car is already connected as they can link to a smartphone for emergency road assistance, register real-time traffic alerts.

Approximately 1.35 million people are cut short of their lives as a result of a road traffic crash. This is mostly due to not following the traffic rules despite various actions taken by the traffic police department. One of the ways to overcome this challenge is by developing autonomous cars. Autonomous cars use sensors and image processing algorithms to detect the surroundings commute and uses road sign and pedestrian detection to make the ride safer and thus following all the traffic rules which in turn reduces road traffic disasters.

\section{LITERATURE SURVEY}

The initial trials on the automated driving system began around 1950s. The first semi-automated car was developed in 1977 by a Japanese Laboratory, this model required specially marked streets and the computing was done on an analogue computer. The first truly autonomous car appeared in the 1980s which was funded by DARPA and developed by Carnegie Mellon University's Navlab and ALV Projects. Turk, Moregenthaler, Gremban, Marra et al. (1988) The ALV car achieved speeds of $31 \mathrm{kmph}$ on two-lane roads, with obstacle avoidance and off-road driving in day and night. From then on over the years many companies and organisations have developed various prototypes.

The Society Of Automotive Engineers et al. (2018) in their article classify the Driving Automation into 6 different levels based on driver intervention and attentiveness required, rather than the vehicle capabilities, although they are very closely related. These levels are:

- Level 0: No Automation, Manual Control. Human performs all driving tasks.

- Level 1: Driver Assistance, the vehicle features a single automated system.

- Level 2: Partial Automation, the vehicle can control steering and acceleration.

- Level 3: Conditional Automation, environmental detection capabilities are included.

- Level 4: High Automation, the vehicle performs all driving tasks under specific conditions.

- Level 5: Full Automation, the vehicle performs all driving tasks under all conditions. Zero human interaction.

\section{CHALLENGES}

Autonomous Driving makes a quantum shift to the future, where the driver will have smaller or no role at all in driving his or her vehicle. Barabás, Todorut, Cordos, Moleae et al. (2017) indicates the various challenges in in this domain. However, technologies are facing various challenges such as the car must make a faster decision in very diverse condition, in case of many types of imminent accident management there are many concerns about the ability to make the right decision. Other technological challenges are as follows: 
- Artificial Intelligence is still not able to function properly in a chaotic inter-city environment.

- Susceptibility of the car's sensing and navigation systems to different types of weather.

- A car's computer could potentially be compromised along with its communication.

- Current Road infrastructure may need changes for automated cars to function optimally.

\section{OBJECTIVES}

With the motive to build the Ghost Car as shown in Fig 1, we aim to add the following autonomous features to our project:

- Road Sign and Obstacle Detection System(RSOD): This system assists in determining various road signs and obstacles and act accordingly. The obstacle detection system uses the in-built algorithm to detect an obstacle on the road using minimal vehicle parameters.

- Lane Keep Assistance (LKA): This is the most unique feature of the project and it helps the car to stay within the lane boundaries with or without the lane markings on the road. The in-built algorithm roughly calculates the width of the road and then decides to keep the car on one side of the road at any given time.

- Traffic Jam Assistance (TJA): This system decides when to overtake slower moving vehicles. It helps the driver to arrive more relaxed at their destination, even though the dense traffic. As a partially automated comfort function, the system takes over the longitudinal and lateral guidance of the vehicle. This means that the car can drive off, accelerate and brake automatically, as well as steer the vehicle within certain constraints.

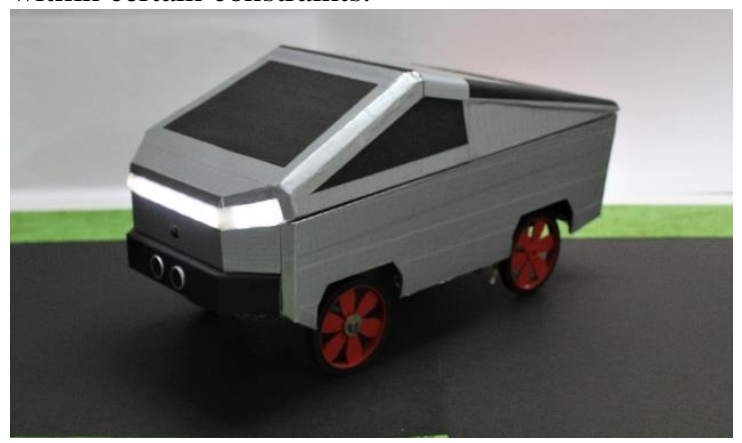

Fig 1: Ghost Car Prototype.

\section{IMPLEMENTATION}
A. CNN TRAINING FOR AUTONOMOUS DRIVING

The Ghost Car determines the steering angle based on its current position and the pathway available for it to travel. A Convolutional Neural Network Model as shown in Fig 2, was trained to map each pixel which was given as input from the camera that was placed on the bumper of the car to one of the pre-defined steering commands i.e. turn left or turn right or go straight. As Maruisz et al. (2016), indicates the efficiency of end to end approach in their work. This end-to-end approach proved powerful as with minimum training data the CNN model learned to drive on roads with or without lane markingss.

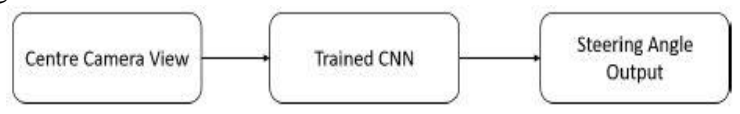

Fig 2: CNN Model

A video recorded from the camera when the car was driven manually on the track was used as training data. This video was then broken down into frames with each frame being mapped to one particular steering angle that would enable the car to move in a direction so that it stays within the lane. These frames along with their respective steering angle were fed into CNN training model. While training the CNN we selected only the data where the car was staying within the lane. Once the data with the final set of frames were selected, this data was augmented in various ways to teach the model to respond accordingly to various situations as shown in Fig 3. Thus, CNN would predict an output from one of the 3 nodes in the output layer.
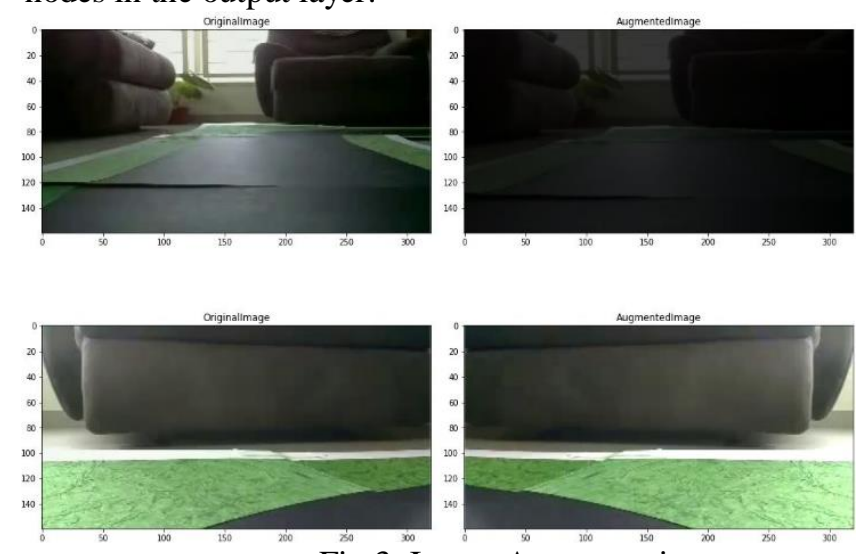

Fig 3: Image Augmentation

\section{B. REALTIME OBJECT DETECTION USING YOLO}

To detect objects such as persons, cars, trucks, animals, traffic lights etc., 'You Only Look Once'(YOLO) algorithm was used. YOLO can be implemented both on CPU or GPU. Although, the implementation of YOLO on CPU is easier, but the efficiency of the algorithm to detect objects in terms of frames per second is slow. Hence, we took up the task of implementing YOLO on GPU which helped us to achieve much higher efficiency. It detected objects as fast as 20-22 frames per second, while YOLO on CPU ran at 0.5-0.8 frames per second. 


\section{International Journal of Engineering Applied Sciences and Technology, 2020 \\ Vol. 5, Issue 3, ISSN No. 2455-2143, Pages 170-173 \\ Published Online July 2020 in IJEAST (http://www.ijeast.com)}

\section{TRAFFIC SIGN DETECTION USING CNN}

For Traffic Sign Detection, we used German Traffic Sign dataset which comprised of 43 different traffic signs, with each traffic sign having images of $32 \times 32$ pixel. Before passing these images as input to CNN training model, all the images were preprocessed. The preprocessing of images took place in 3 steps. Firstly, the image which would be in RGB colour space would be converted to Gray colour space. Secondly, then the histogram of the image would be equalized, this allows for areas of lower local contrast to gain higher global contrast. Thirdly, rescaling of the image would be done to transform every pixel value from range $[0,255]$ to range $[0,1]$, which will treat all images in the same manner and contribute evenly to the total loss. This preprocessed image is fed as input to the $\mathrm{CNN}$ training model which would predict an output from one the 43 classes as shown in Fig 4 .

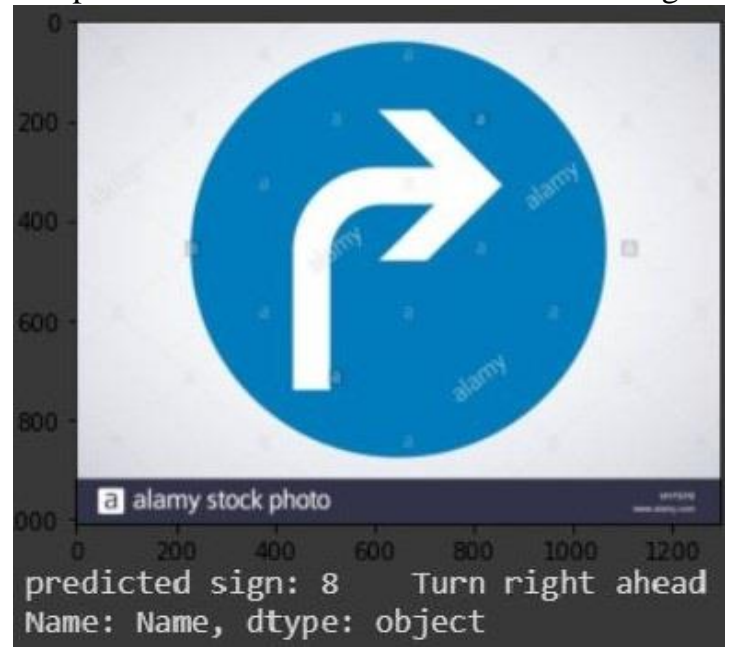

Fig 4: Traffic Sign Detection.

\section{ACTUATION IN PROTOTYPE MODEL}

The model car was designed to perform forward and backward movement through rear-wheel-drive chassis design, along with front-wheel steering up to an angle of $25^{\circ}$ on both left and right directions as shown in Fig 5. The track design was an oval track (as shown in Fig 6) which can be detached and rearranged to form different designs for testing and training the CNN model. The output from above 3 Sections was fed as input to the prototype's actuation code, which would then take an appropriate decision on the car's movement.

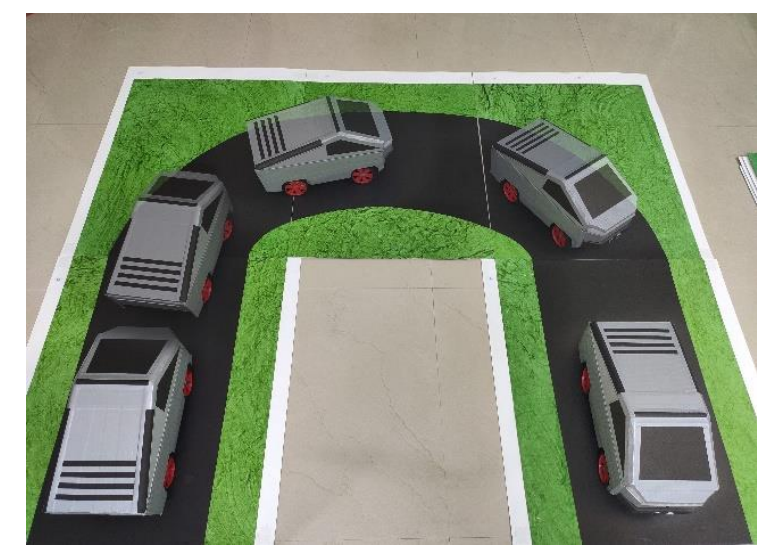

Fig 5: Prototype Turning at angle of $25^{\circ}$ to right.

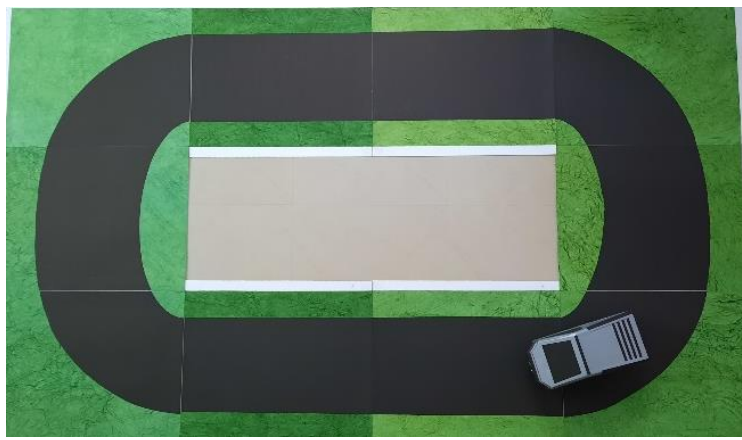

Fig 6: Track Model

\section{CONCLUSION}

The Ghost Car is a prototype built to mimic a level 5 Driving Automation that can drive in conditions where lane markings are not available. It detects traffics signs and various objects and it can take appropriate decisions in the most adverse conditions.

\section{ACKNOWLEDGEMENT}

We would like to thank our friends for extending help, our family for keeping us motivated. Finally, we would like to show our gratitude to Mr. Shantakumar, our guide for guiding us along the project.

\section{REFERENCES}

1. Mariusz Bojarski, Davide Del Testa, Daniel Dworakowski. (2016) End to End Learning for Self-Driving Cars, by NVIDIA Corporation Holmdel, NJ, pp.4-6. https://arxiv.org/abs/1604.07316.

2. SAE International. (2014), Taxonomy and Definitions for Terms Related to On-Road Motor Vehicle Automated Driving Systems, pp 1-2, URL: http://standards.sae.org/j3016_201401. 
3. Society of Automotive Engineers (SAE). (2018). Taxonomy And Definitions For Terms Related To Driving Automation Systems For On-Road Motor Vehicles, p.1, https://blog.ansi.org/2018/09/saelevelsdriving-automation-j-3016-2018/\#gref.

4. I Barabás A Todoruț, N Cordoș, A Moleae. (2017) Current challenges in autonomous driving, by Department of Automotive Engineering and Transports, Cluj-Napoca, Romania, pp.2-4, DOI: $10.1088 / 1757-899 X / 252 / 1 / 012096$

https://iopscience.iop.org/article/10.1088/1757$\underline{899 X / 252 / 1 / 012096 .}$

5. K. Bimbraw. (2015) "Autonomous cars: Past, present and future a review of the development12th International Conference on Informatics in Control, Automation and Robotics (ICINCO), Colmar, 2015, pp. 191-198. https://ieeexplore.ieee.org/document/7350466.

6. M. G. Bechtel, E. Mcellhiney, M. Kim and H. Yun, (2018), "Low-Cost Deep Neural Network-Based Autonomous Car," IEEE 24th International Conference on Embedded and Real-Time Computing Systems and Applications (RTCSA), pp. 11-21, DOI: 10.1109/RTCSA.2018.00011. https://ieeexplore.ieee.org/document/8607229.

7. A. R. Fayjie, S. Hossain, D. Oualid and D. Lee, (2018) "Driverless Car: Autonomous Driving Using Deep Reinforcement Learning in Urban Environment," 15th International Conference on Ubiquitous Robots (UR), 2018, pp. 896-901, DOI: 10.1109/URAI.2018.8441797.

https://ieeexplore.ieee.org/document/8441797.

8. C. Pozna and C. Antonya, (2016) "Issues about autonomous cars," IEEE 11th International Symposium on Applied Computational Intelligence and Informatics (SACI), Timisoara, 2016, pp. 1318, DOI: $\quad 10.1109 /$ SACI.2016.7507360. https://ieeexplore.ieee.org/document/7507 360.

9. T. Srivastava, P. Arora, C. Wang and S. Chattopadhyay, "Work-in-Progress: Road ContextAware Intrusion Detection System for Autonomous Cars," 2018 International Conference on Embedded Software (EMSOFT), Turin, 2018, pp. 1-3, DOI: 10.1109/EMSOFT.2018.8537210. https://ieeexplore.ieee.org/document/8537210.

10. Strigl, Daniel \& Kofler, Klaus \& Podlipnig, Stefan. (2018). Performance and Scalability of GPU-Based Convolutional Neural Networks. pp.317-324. DOI:10.1109/PDP.2010.43.

https://www.researchgate.net/publication/2213922
98 Performance and Scalability of GPUBased_Convolutional_Neural_Networks

11. Shao, Xinqing Wang, Fanjie Meng, and Jian Tang. (2018). Real-Time Traffic Sign Detection and Recognition. Pp.10-13, DOI: 10.3390/s18103192 https://www.ncbi.nlm.nih.gov/pmc/articles/PMC6 $\underline{210476 /}$

12. C. Wang, (2018) "Research and Application of Traffic Sign Detection and Recognition Based on Deep Learning," 2018 International Conference on Robots \& Intelligent System (ICRIS), pp. 150-152, DOI: 10.1109/ICRIS.2018.00047. https://ieeexplore.ieee.org/document/8410256

13. Howal, Sadanand \& Jadhav, Aishwarya \& Arthshi, Chandrakirti \& Nalavade, Sapana \& Shinde, Sonam. (2020). Object Detection for Autonomous Vehicle Using TensorFlow. DOI:10.1007/978-3030-30465-2_11.

https://www.researchgate.net/publication/3366708 46_Object_Detection_for_Autonomous_Vehicle_ Using_TensorFlow

14. M.A Turk, D.G Moregenthaler, K.D Gremban, M Marra. (1988). VITS- a vision system for automated land vehicle navigation, pp. 342-361, DOI:10.1109/34.3899.

https://ieeexplore.ieee.org/document/3899

15. Song, Rui \& Wetherall, Jon \& Maskell, Simon \& Ralph, Jason. (2020). Obstacle Detection for Autonomous Car. 331-341. DOI: $10.5220 / 0009354503310341$. https://www.researchgate.net/publication/3414658 32_Weather_Effects_on_Obstacle_Detection_for Autonomous_Car/ 